\title{
The elusive muscular dystrophy gene. Fifth Muscular Dystrophy Group Workshop on the X chromosome and muscular dystrophies, April 1986
}

In spite of intensive research on Duchenne muscular dystrophy, the cause of this disease, and the related Becker muscular dystrophy, continues to elude scientists. This was the subject of a meeting organised by the Muscular Dystrophy Group of Great Britain held recently at the Royal Society in London.

Peter Lachmann introduced the sessions, highlighting the optimism that was felt over a year ago when Lou Kunkel identified a sequence (pERT87) which is deleted in $7 \%$ of DMD patients. ${ }^{1}$ Surprisingly, pERT 87 has so far not been found to be part of a coding sequence and the locus recombines with the DMD mutation in $5 \%$ of meioses. Although this was a setback, Ed Southern emphasised that significant progress had been made over the last three years since the genetic linkage studies had localised both DMD and BMD to a very small region of $\mathrm{Xp} 21$. This had resulted in the first prenatal diagnosis of DMD. ${ }^{2}$ However, as the meeting progressed it became clear that the data indicate that the DMD locus is quite unlike any disease locus yet examined.

Ron Worton, from Toronto, presenting the second breakthrough, described how his group had succeeded in cloning the $X ; 21$ translocation breakpoint in the female patient suffering from the disease. ${ }^{3}$ This junction fragment (XJ1.1) is deleted in a small number of patients and, like pERT87, it recombines with the DMD locus in approximately $5 \%$ of the meioses. Interestingly, not all the patients deleted for pERT87 are deleted for XJ1.1 and vice versa. Martin Bobrow reported data from his group and the analysis of their deletions by Lou Kunkel. The deletions are as small as $\mathbf{4 5}$ kilobase pairs $(\mathrm{kb})$ and as large as several thousand $\mathrm{kb}$ of DNA. He also noted that one Becker patient was deleted for the pERT87 probe but not at the XJ1.1 locus. Thus, although the $\mathrm{XJ} 1.1$ probe was derived from a translocation that results in a Becker like phenotype, it is clearly not the 'Becker locus' as opposed to pERT87 being the 'DMD locus'. The deletions reported by Martin Bobrow appeared to be contiguous. In one patient, Bobrow's group noticed that one of the pERT87 sequences hybridised to a band which was smaller in several enzyme digests than the normal band. This same patient was normal at the XJ1.1 locus and at pERT87 loci lying distal to the sequence revealing the deletion. This boy must therefore have a deletion starting within this pERT87 sequence and extending towards the $\mathrm{XJ} 1.1$ locus. The determination of the extent of this deletion might give some insight into the physical distance between XJ1.1 and pERT87.

Genetic studies with these new probes also reveal surprising segregation patterns. Almost every laboratory reported an instance of a crossover between the pERT87 locus and the DMD or BMD mutation. In those families where recombination has been observed, which are also informative for other RFLPS on the centromere side of the locus, the loci pERT87 and XJ1.1 segregate together and map the mutation in these families distal to the probes. However, Ron Worton presented data in a family where the crossover had occurred with XJ1.1 but not with pERT87, suggesting a different location of the mutation in this family from the ones previously studied. He suggested caution in assigning a particular position for the mutation as this might be different in different families. One of the recombination events reported by Worton actually occurred within the XJ1.1 locus itself. Peter Harper also presented the Cardiff data with pERT87, which included a recombinant within the pERT87 locus. In one BMD family there appeared to be three recombinants with the pERT87 locus, although it was suggested that this might not be BMD but another similar dystrophy.

In spite of the high recombination rate around and within the locus, Bert Bakker from Peter Pearson's group in Leiden demonstrated that it is still feasible to use the DNA probes for diagnostic purposes by analysing bracketing markers. They used several markers simultaneously to determine the haplotype of the DMD chromosome and define the crossovers between the various loci. If crossovers were observed between markers flanking the mutation it was 
reasonably supposed that further recombination was unlikely.

The order of probes was defined physically using somatic cell hybrids by Yvonne Boyd and her colleagues from Oxford. They presented data which indicated the order Xpter-pERT87-XJ1.1-754-Xcen. This work demonstrated that the breakpoints in the female patients with translocations are different and may span several thousand $k b$ of DNA sequence. The minimum distance between XJ1.1 and pERT87 was estimated to be $170 \mathrm{~kb}$ but it could be much larger than this.

The observed recombination rate and the heterogeneity in the female breakpoints which give rise to DMD and BMD could be explained in several ways. The hypotheses put forward included the involvement of a very large gene, several genes, or the occurrence of chromosomal inversions in the region of the mutations. Ed Southern pointed out that chromosomal changes in one region of Xp21 might alter the long range chromosomal structure and thus affect gene expression in another part of Xp21.

Susan Forrest and Susan Kenwrick in my own laboratory presented data on the mapping of sequences between breakpoints in several patients suffering from DMD, glycerol kinase deficiency, and adrenal hypoplasia. One patient was not deleted for $\mathrm{XJ} 1.1$ or pERT87, thus placing the mutation distal to . both of these probes in this particular case.

One of the newer technologies being applied to the problem of gene arrangements is that of pulsed field electrophoresis. Susan Kenwrick presented preliminary studies of the Xp21 region using this method. This technique enables the mapping of sequences over several hundred $\mathrm{kb}$ of DNA rather than up to $20 \mathrm{~kb}$ of DNA using conventional electrophoretic separation methods. She suggested that in view of the complexity of the region, it was also wise to analyse the order of probes in the normal population as well as in patients. Ed Southern's group presented a strategy using this technique to analyse the region using Ron Worton's probe as a starting point. Gert jan van Ommen from Leiden presented his results with pERT87 and deduced from his PFG analyses that the DMD region might encompass as much as $2500 \mathrm{~kb}$ or more. This technique promises to give us much more useful information in the future.

Nick Thomas, from Peter Harper's group, presented data on another DMD patient with glycerol kinase deficiency and adrenal hypoplasia with a visible deletion. This patient is deleted for 754 , $\mathrm{XJ} 1.1$, pERT87, and the distal marker C7 but not 996. However, he does not suffer from chronic granulomatous disease, as did the patient BB used by Kunkel to clone the pERT probes. Malcolm Ferguson-Smith presented a DMD patient with a deletion which was estimated to be approximately $6000 \mathrm{~kb}$ from flow cytometry studies. This patient was deleted for 754, pERT87, XJ1.1, but not C7, and expressed the Kell blood group antigen. In fact, from the flow cytometry experiments, he showed a deletion of a similar size to that found in BB's X chromosome although he showed a different clinical phenotype. Douglas Wilcox from the same group showed how this deletion was segregating in the family and how useful the flow cytometer can be in the determination of the presence of deletions in females at risk of being carriers. He also noted that the DMD gene could have found itself in a region of high recombination frequency rather than itself being causative of this effect.

In summary, over the last few years the molecular analysis of DMD and BMD using DNA markers has done much for the diagnosis of these disorders. The next year promises to be full of surprises as the scientists begin to unravel the complexities of the molecular basis of these diseases.

\section{Note added in proof}

Recently, highly conserved sequences in the region of the pERT87 probes have been identified by Kunkel and colleagues. This has led to the isolation of a candidate gene sequence for this disease.

\section{KAY E Davies MRC Senior Research Fellow, Nuffield Department of Clinical Medicine, John Radcliffe Hospital, Oxford OX3 9DU.}

\section{References}

1 Monaco AP. Bertelson CJ, Middlesworth W. et al. Detection of deletions spanning the Duchenne muscular dystrophy locus using a tightly linked DNA segment. Nature 1985;316:842-5.

2 Bakker E, Hofker MH, Goor N, et al. Prenatal diagnosis and carrier detection of Duchenne muscular dystrophy with closely linked RFLPs. Lancet 1985;i:655-8.

3 Ray PN, Belfall B, Duff C, et al. Cloning of the breakpoint of an $\mathrm{X} ; 2$ I translocation associated with Duchenne muscular dystrophy. Nature 1985;318:672-5. 